\title{
Lactobacillus pentosus S-PT84 and Rubus suavissimus leaf extract suppress lipopolysaccharide-induced gut permeability and egg allergen uptake
}

\author{
Hiroaki Kanouchi ${ }^{1,2}$, Kaustav Majumder $^{1,3}$, Hiroshi Shibata ${ }^{4}$ and Yoshinori Mine ${ }^{1 *}$ (1)
}

\begin{abstract}
Increased gut permeability facilitates the uptake of food allergens into the bloodstream and triggers allergenic reactions. The present study aimed to evaluate the effect of Lactobacillus pentosus S-PT84 (S-PT84) and Rubus suavissimus leaf extract (RSLE) against egg ovomucoid (OVM)-uptake in lipopolysaccharide (LPS)-induced increased gut-permeability mice model of food allergy. Six-eight weeks old, female C57BL6 mice were continuously fed with LPS (300 $\mu \mathrm{g} / \mathrm{kg}$ BW), for 3 months to increase gut permeability. Reduction in the expression of sealing claudin-4, increase in the expression of pore-forming claudin-2, and increase in D-mannitol absorption into the blood plasma in the LPS treated groups suggested the increase in gut permeability after LPS treatment. The oral administration of major egg allergen, OVM, after LPS intervention, significantly increased the plasma mast cell protease-1 and OVMspecific IgE compared to the negative control group. These results indicated that continuous LPS intervention developed OVM-induced food allergy. However, both the treatment of S-PT84 and RSLE suppressed the claudin-2 expression and the gut permeability induced by LPS. Furthermore, S-PT84 and RSLE treatment also reduced the plasma mast cell protease-1 and OVM-specific IgE, indicating the potential beneficial effect against LPS intervention developed OVM-induced food allergy. These findings suggest that S-PT84 and RSLE ameliorated LPS induced gut permeability and food allergic reactions.
\end{abstract}

Keywords: Gastro-intestinal tract, Gut-permeability, Food allergy, Tight junction, Claudin-2, Lipopolysaccharide, Ovomucoid, Rubus suavissimus, Lactobacillus pentosus

\section{Introduction}

Intestinal epithelial cells tightly bind to the neighboring cells and forms tight junctions (TJs), TJs primarily acts as a barrier between the luminal environment of the gastrointestinal tract, and the internal environment of the body, the bloodstream (Furuse 2010). TJs also selectively absorbs and secretes nutrients, solutes, and water-soluble molecules across the barrier. The TJs in the intestinal surface also acts as a protective barrier for pathogens, toxins, and allergens. Proteins (claudins (Cld), ZO proteins, occludin, and tricellulin) present in the TJs have dynamic structure and actively remodeled during a variety of conditions of

\footnotetext{
*Correspondence: ymine@uoguelph.ca

${ }^{1}$ Department of Food Science, University of Guelph, Guelph, Ontario N1G 2W1, Canada

Full list of author information is available at the end of the article
}

the intestine (Furuse 2010; Garcia-Hernandez et al. 2017). Cld-1, -2, -3, -4, -5, -8, -9, -10, -11, -12, -13, -14, -15, -17, and -18 are detected in mouse intestine using reverse transcriptase PCR. Among of them, Cld-2, 3, 7, and 15 are highly expressed in the intestine. Almost all Clds serves sealing, while Cld-2 and -15 make channels for ion and water transport. Therefore, increase of leaky Cld-2 and -15 in tight junction elevates intestinal permeability. Disturbance of the expression of tight junction related protein causes leaky gut condition.

It has been reported that various factors such as stresses, diet, dysbiosis, and drugs increase gut permeability (Ukena et al. 2007; Zhang et al. 2011; Singh et al. 2017; Cho \& Song 2018). There is a possibility that a significant increase in the intestinal permeability occurs without any subjective symptoms. Increasing intestinal permeability, toxins, antigens, 
and bacteria could enter the bloodstream (Mu et al. 2017). These molecules are generally blocked by the intestinal barrier to enter the bloodstream. If these molecules enter the bloodstream, they become antigens resulting in an allergic response. Increased gut permeability could be one of the risk factors of possible food allergy.

Lipopolysaccharide (LPS) is a major inflammatory molecule exist in Gram-negative bacterium. LPS binds to toll like receptor-4 (TLR-4) and activates signaling pathway of inflammation (Lu et al. 2008), inhibiting proliferation, and inducing apoptosis $\mathrm{Lgr}^{+}$intestinal stem cell (Neal et al. 2012). Recently, Wassenaar and Zimmermann summarized about LPS in food, food supplements, and probiotics (Wassenaar \& Zimmermann 2018). Large number of Gramnegative bacteria exists in colon and produces LPS. Their estimate amount of LPS is between 2 and $50 \mathrm{mg}$ in human colon. However, the colonic LPS is not harmful, as it does not reach cell surface due to the presence of mucus layer. Orally administrated LPS seems to be also nontoxic, because the amount is too small compared to that derived from bacteria in the colon.

Since abnormal intestinal inflammation is a key event of the gut barrier disturbance, anti-inflammatory molecule could prevent the impaired barrier function. It has been reported that different food-derived phytochemicals suppress inflammatory responses via modulating the Nuclear Factor kappa B (NF- $\mathrm{kB})$ signaling pathway and inhibiting the production of pro-inflammatory cytokines (Andrade \& Valentão 2018). Among the phytochemicals, teapolyphenols such as catechin and the derivatives are recognized for their anti-inflammatory activity. Excess production of reactive oxygen species also promotes inflammation through increased production of proinflammatory cytokines. Anti-oxidative effects of phytochemicals are also expected to exhibit anti-inflammatory effects. Catechin could exhibit potent anti-oxidative effect and regulate vital signaling pathways of inflammation $(\mathrm{Oz}$ 2017). Trilobatin identified in Rubus suavissimus leaf extract (RSLE) is one of the flavonoids and attenuates LPSmediated inflammatory response via suppression of NF- $\mathrm{kB}$ signaling pathway in RAW 264.7 macrophage (Fan et al. 2015). Moreover, it is well known that the dysbiosis of gut microbiota is closely related to chronic inflammationrelated diseases (Tsai et al. 2019). Earlier studies have demonstrated that intervention of probiotics and prebiotics could also provide health beneficial effect against chronic inflammation-related diseases by improving the bacterial dysbiosis or by producing short chain fatty acids (RíosCovián et al. 2016; Liu et al. 2018). Maekawa et al. (2016) reported that heat killed Lactobacillus pentosus S-PT84 (SPT84) suppresses gastric inflammation in a murine gastrointestinal candidiasis model.

Therefore, in the present study, we evaluated the effect of continuous oral administration of LPS in modulating gut permeability, and the potential risk of increase in allergen uptake, resulting in food allergy. The effects of SPT84 and RSLE on the intestinal barrier disturbance and prevent allergic reaction were examined.

\section{Material and methods \\ Chemicals and reagents}

LPS isolated from Escherichia coli (E. coli) O111: B4 was purchased from Sigma (Oakville, ON, Canada). Ovomucoid (78\%) was purchased from Neova Technologies (Abbotsford BC, Canada). Stock LPS solution $(150 \mu \mathrm{g} / \mathrm{mL})$ was prepared in autoclaved water and kept at $-20^{\circ} \mathrm{C}$ for further usage. D-mannitol was purchased from Sigma. RSLE and S-PT84 were provided from Marugen Pharmacy (Okayama, Japan) and Suntory Wellness Ltd. (Kyoto, Japan) respectively. S-PT84 was cultivated in a medium containing glucose and yeast extract (AromildTM, SK yeast extract $\mathrm{Hi}-\mathrm{K}$ ) at $37^{\circ} \mathrm{C}$ for $24 \mathrm{~h}$. Cultured bacteria were collected by centrifugation at $9190 \mathrm{x} \mathrm{g}$ for $5 \mathrm{~min}$, washed twice with sterile saline and once with distilled water, and heat-killed at $95^{\circ} \mathrm{C}$ for $5 \mathrm{~min}$. S-PT84 was lyophilized and then used in this study. Experimental foods containing S-PT84 were prepared by ENVIGO (Madison, WI). Low dose $(0.06 \% \mathrm{w} /$ w) and high dose $(0.6 \% \mathrm{w} / \mathrm{w})$ were supplemented as show in Additional file 1: Table S1. The RSLE used in the present study contains $30.1 \%$ polyphenol and $7.0 \%$ glucose oxidase type polyphenol. Through the chromatography analysis, the gallic acid (GA) (peak1) $(1659.0 \pm 142.5 \mathrm{mg} /$ $100 \mathrm{~g}$ DW), ellagic acid (EGA) (peak2) $(4622.7 \pm 142.0 \mathrm{mg} /$ $100 \mathrm{~g} \mathrm{DW})$, and rubusoside (RUB) (peak3) $(333.0 \pm 67.7$ $\mathrm{mg} / 100 \mathrm{~g} \mathrm{DW}$ ) were mainly identified in RSLE as shown in Additional file 2: Figure S1. Low dose-(0.5\%) and high dose-RSLE $(1.0 \%)$ containing drinking water were fleshly prepared every day during the study.

\section{Animal experiment}

Seventy-two female C57BL6 mice (6-8 weeks, 18-20 g) were purchased from Charles River Laboratories (Montreal, $\mathrm{QC}$ ). Mice were housed on a $12 \mathrm{~h} \mathrm{light/dark}$ cycle and permitted ad libitum access to water and normal chew or experimental diet. Summary of animal experiment was shown in Fig. 1 and diet formulation for PT-84 was shown in Additional file 1: Table S1. Mice were randomly divided into 6 groups ( $n=12$ mice/group). Mice were treated with experimental diets for 2 weeks before LPS feeding. LPS $(1.5 \mu \mathrm{g} / \mathrm{mL})$ containing water was prepared using stock LPS solution. The water changed twice every week. Combination of drinking water and food was as follow: water and control diet in negative control group (NC); LPS water and control diet in LPS group (LPS); LPS water and 0.06\% S-PT84 in LPS-probiotic low dose group (LPS-BL); LPS water and $0.6 \%$ S-PT84 in LPS-probiotic high dose group (LPS-BH); LPS-0.5\% RSLE water and control diet in LPStea extract low dose group (LPS-TL); LPS-1.0\% RSLE water 


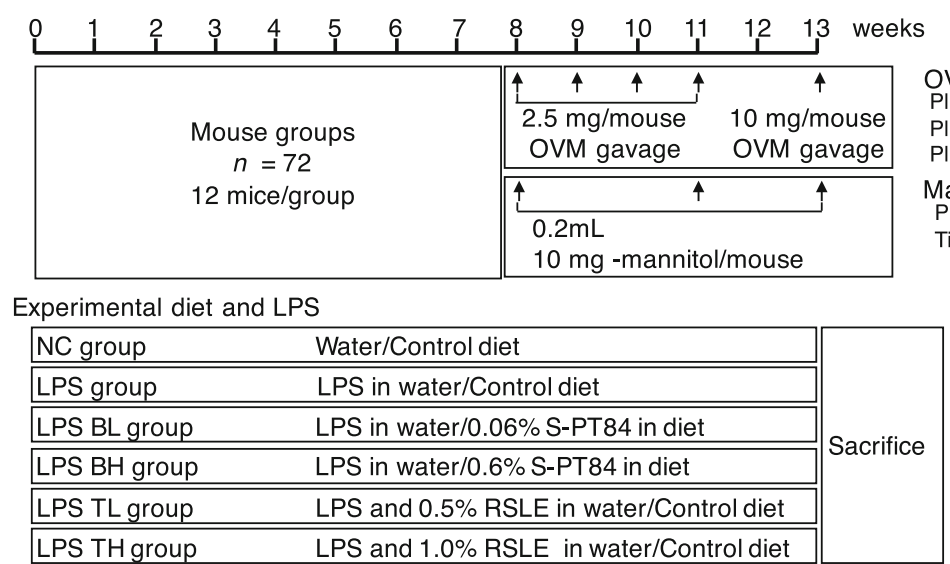

Fig. 1 Outline of the animal experiment design. Mice were divided into 6 groups ( $n=12$ mice/group). Mice were fed S-PT84 or RSLE for 13 weeks. LPS containing water $(1.5 \mu \mathrm{g} / \mathrm{mL})$ fed for 13 weeks excepting negative control group. The half of each group $(n=6)$ was fed ovomucoid (OVM) 4 times at 8, 9, 10, and 11 weeks except negative group, respectively. At 13 weeks, the mice were challenged orally with OVM (10 mg/ mouse). Two hour later mice were sacrificed for collection of blood. The other half of mice were $(n=6)$ fed D-mannitol at 8,11 and 13 weeks, and $2 \mathrm{~h}$ later of end point, mice were sacrificed for collection of blood and intestine

and control diet in LPS-tea extract high dose group (LPSTH). Mice in each group were divided into two following subgroups OVM treat-subgroup and D-mannitol treatsubgroup. In OVM treat-subgroup, mice were gavaged with $0.2 \mathrm{~mL}$ OVM solution (adjusted to $2.5 \mathrm{mg} /$ mouse) at $8,9,10$ and 11 weeks. Then, at 13 weeks, mice were gavaged with $0.2 \mathrm{~mL}$ OVM solution (adjusted to $10 \mathrm{mg} /$ mouse) and after $2 \mathrm{~h}$, mice were sacrificed. In D-mannitol treat-group, all mice were gavaged with $0.15 \mathrm{~mL}$ Dmannitol solution $(0.6 \mathrm{~g} / \mathrm{kg} \mathrm{BW}$ D-mannitol $)$ at 13 weeks, and at $2 \mathrm{~h}$ after gavage, mice were sacrificed. All mice were sacrificed in $\mathrm{CO}_{2}$ filled box, and blood was immediately collected via cardiac puncture. The blood was transferred into EDTA-tube and then centrifuged to obtain plasma. Ileum was collected and transferred the tubes filled with RNAlater Stabilization Solution (Thermo Fisher Scientific, Mississauga, ON, Canada). The animal study was approved by the University of Guelph Animal Care Committee and was performed in accordance with the Guide to the Care and Use of Experimental Animals from the Canadian Council on Animal Care (Olfert et al. 2017). The Animal Utilization Protocol (AUP) number for the animal study is AUP e3502. The mice were housed in the Central Animal Facility (CAF) at the University of Guelph (Guelph, ON) for the duration of study.

\section{Gut permeability}

FITC-labelled OVM was prepared using NHSFluorescein (Thermo Fisher Scientific) according to the manufacturer's instructions. Briefly, $78 \mathrm{mg}$ NHS-FITC dissolved in $78 \mu \mathrm{L}$ DMSO added to $15 \mathrm{~mL}$ OVM $(20 \mathrm{mg} /$ $\mathrm{mL}), 4.5 \mathrm{~mL}$ borate buffer $(0.1 \mathrm{M}, \mathrm{pH} 8.5)$, and $2.2 \mathrm{~mL}$ water and incubated for $2 \mathrm{~h}$ on ice. Then, the FITClabelled OVM was dialyzed in water $(2 \mathrm{~L})$ using dialysis tube (Fisher brand Regenerated Cellulose Dialysis Tubing, MWCO 6000-8000, Thermo Fisher Scientific) for 12 days in dark cold room, water changed twice every day. The concentration of FITC-labelled OVM was measured using BCA Protein Assay (Thermo Fisher Scientific). The protein concentration was $13 \mathrm{mg} / \mathrm{mL}$. Absorbance of $280 \mathrm{~nm}$ and $493 \mathrm{~nm}$ were measured. Labeling efficiency was calculated as follow: Labeling efficiency $=\mathrm{A} 493 / \varepsilon_{\text {fluor }} \times \mathrm{Abs}_{280^{-}}(\mathrm{A} 493 \times$ correction factor $)$ $/ \varepsilon_{\text {protein }}$. Correction factor $=0.3, \varepsilon_{\text {fluor }}=70,000, \varepsilon_{\text {protein }}=$ OVM extinction coefficients $\left(10,065 \mathrm{M}^{-1} \mathrm{~cm}^{-1}\right)$. As a result, the labeling efficiency was $81.9 \%$. At 8 and 11 weeks, 6 mice in all group were force fed $0.2 \mathrm{~mL}$ the FITC-labelled OVM (adjusted to $2.5 \mathrm{mg} /$ mouse) instead of non-labeled OVM. After $2 \mathrm{~h}$, blood was collected from saphenous vein and collected into EDTA tube to prepare plasma sample. Fifty $\mu \mathrm{L}$ saturated ammonium sulfate was added to $50 \mu \mathrm{L}$ plasma and the sample was stood for $20 \mathrm{~min}$ at room temperature. Pellet after centrifuge $(10,000 \mathrm{~g}$ for $5 \mathrm{~min})$ was rinsed well in $50 \%$ ammonium sulfate. The sample were centrifuged again (10, $000 \mathrm{~g}$ for $5 \mathrm{~min}$ ), then the pellet was dissolved in $50 \mu \mathrm{L}$ water. Fluorescent intensity was measured using Synagy HTX (excitation, 494 nm; emission, $518 \mathrm{~nm}$; BioTek, Winooski, VT, USA). To make standard curve of plasma OVM concentration, the fluorescent intensity was measured $50 \mu \mathrm{L}$ FITC-labelled OVM $(1.3,13,130$, and 1300 $\mathrm{ng} / \mathrm{mL}$ in mouse plasma) precipitated with $50 \%$ ammonium sulfate as same method and measured the fluorescent intensity.

At the end point, 6 mice excepting OVM fed mice were force fed $0.2 \mathrm{~mL}$ D-mannitol solution (adjusted to $14 \mathrm{mg} /$ mouse). After $2 \mathrm{~h}$, blood was collected and plasma was prepared to measure D-mannitol concentration 
using commercial kit (D-mannitol Colorimetric Assay kit, Sigma-Aldrich, St. Louis, MO, USA).

\section{Real time-PCR}

Total RNA was extracted and purified using Aurum total RNA Mini Kit (Bio-Rad, Mississauga, ON, Canada). Total RNA concentration was determined using a NanoDrop 8000 (Thermo Fisher Scientific). Total RNA was reversetranscribed using Maxima First Strand cDNA Synthesis Kit for RT-qPCR, with dsDNase (Thermo Fisher Scientific). RT-PCR was done using MyiQ Real-Time PCR Detection Systems (Bio-Rad). A list of primer sequence was shown in Additional file 3: Table S2. Relative gene expression was calculated using the $2^{-\Delta \Delta \mathrm{Ct}}$ method (Bookout \& Mangelsdorf 2003) using GAPDH as the reference gene.

\section{ELISA}

Plasma MCPT-1 concentration was measured using eBioscienc Mouse MCPT-1 (mMCP-1) ELISA Ready-SET-Go Kit (Fisher Scientific) according to the manufacturer's instructions. Total IgE and specific IgE in plasma was carried out as previously described (Rupa \& Mine 2012).

\section{Statistical analysis}

The data were expressed as mean \pm SEM. Statistical analysis were done using JMP ver. 14 (SAS Institute Inc., Cary, NC). Significant difference was evaluated by unpaired Student's $t$-test. Differences were considered significant if $p$-value $<0.05$.

\section{Results}

\section{Body weight gain and food consumption during the} experiment period

LPS feeding slightly reduced the body weight, however, did not achieve statistical differences (Table 1). The end point of body weight of LPS-BL and LPS-BH groups were significantly high compared to LPS group. Food efficiency of LPS group was lower than that of NC group, but there was no significant difference. LPS-BL, LPS-BH, LPS-TL, LPS-TH, and LPS-BL group showed high food efficiency compared to LPS group.

\section{Measurements of gut permeability}

We evaluated the uptake of OVM into the blood stream at 8 and 11 weeks after LPS intervention. The detection limit of a commercial ELISA kit $(3.12-100 \mu \mathrm{g} / \mathrm{mL})$ for OVM is not sensitive enough to measure the OVM in the blood, thus, we directly measured FITC-labelled OVM. In each period, after $2 \mathrm{~h}$ FITC-labelled OVM $(0.1 \mathrm{mg} / \mathrm{g}$ of body weight) gavage, blood was collected. Plasma OVM concentration did not change among group at 8 weeks (Fig. 2a). At 11 weeks, maximum OVM concentration in NC group was $69 \mathrm{ng} / \mathrm{mL}$, and the average of that was $29.6 \pm 11.0 \mathrm{ng} / \mathrm{mL}$ (Fig. 2b). The averages of OVM were $80.6 \pm 44.5,66.9 \pm$ 25.8, $31.1 \pm 14.0,31.6 \pm 19.6$, and $38.9 \pm 17.2 \mathrm{ng} / \mathrm{mL}$ in LPS, LPS-BL, LPS-BH, LPS-TL, LPS-TH, and LPS-BL group, respectively. There were significant differences in NC, LPS$\mathrm{BH}, \mathrm{LPS}-\mathrm{TL}$, and LPS-TH versus LPS group. LPS feeding seemed to increase gut permeability and RSLE and S-PT84 treatment suppressed its uptake. However, there were big differences of OVM concentration in the same group. We did not conclude LPS feeding for 11 weeks disturbed clearly barrier integrity. At the end point of the experiment (13 week), the plasma D-mannitol concentration was measured after $2 \mathrm{~h}$ oral gavage of D-mannitol $(0.6 \mathrm{mg} / \mathrm{g}$ of body weight, Fig. 3). Each plasma D-mannitol concentration was $2.25 \pm 0.21,3.83 \pm 0.44,3.08 \pm 0.48,2.61 \pm 0.24,3.21 \pm 0.17$, and $2.92 \pm 0.12 \mu \mathrm{M}$ in NC, LPS, LPS-BL, LPS-BH, LPS-TL, LPS-TH, and LPS-BL group, respectively. LPS feeding significantly increased plasma D-mannitol concentration by 1.7 times compared to NC group. Both RSLE and S-PT84 supplementation significantly reduced plasma D-mannitol concentration in dose-dependent manner.

\section{Expression of tight junction related genes and MCPT-1 gene}

Owing to clear the mechanism of the effect of long term LPS feeding on gut barrier function, we measured various kind of $\mathrm{Tj}$ related genes in jejunum from mice that treated with LPS or not (Additional file 4: Table S3). It's seemed that the only Cld-4 expression level was significantly reduced by LPS. The expression was reduced by $35 \%$ in LPS group. Therefore, we focused the Cld-4 expression among all groups (Fig. 4, $n=6$ ). Decreasing expression of Cld-4 by LPS feeding was also found. The relative expression

Table 1 Body weights and food consumptions

\begin{tabular}{lllll}
\hline Group $(\mathrm{n})$ & Initial BW $(\mathrm{g})$ & End point BW $(\mathrm{g})$ & Food consumption/day & Food efficiency $^{\mathrm{a}}$ \\
\hline NC (12) & $17.4 \pm 0.2$ & $23.4 \pm 0.2$ & $2.77 \pm 0.15$ & $23.9 \pm 0.9$ \\
LPS (12) & $17.3 \pm 0.3$ & $22.6 \pm 0.3$ & $2.82 \pm 0.24$ & $20.6 \pm 1.6$ \\
LPS-BL (12) & $16.7 \pm 0.3$ & $24.7 \pm 0.6^{*}$ & $3.15 \pm 0.15$ & $29.5 \pm 1.8^{*}$ \\
LPS-BH (12) & $16.5 \pm 0.3$ & $24.5 \pm 0.6^{*}$ & $3.81 \pm 0.27$ & $23.0 \pm 1.6$ \\
LPS-TL (12) & $16.7 \pm 0.4$ & $22.7 \pm 0.3$ & $2.83 \pm 0.03$ & $23.5 \pm 1.5$ \\
LPS-TH (12) & $17.1 \pm 0.4$ & $23.3 \pm 0.6$ & $3.03 \pm 0.15$ & $22.6 \pm 1.4$ \\
\hline
\end{tabular}

Data were presented as mean $\pm \mathrm{SEM} ;{ }^{*} p<0.05$, vs LPS); ${ }^{a}$ increase BW ( $\left.\mu \mathrm{g}\right) /$ food consumption (g) 

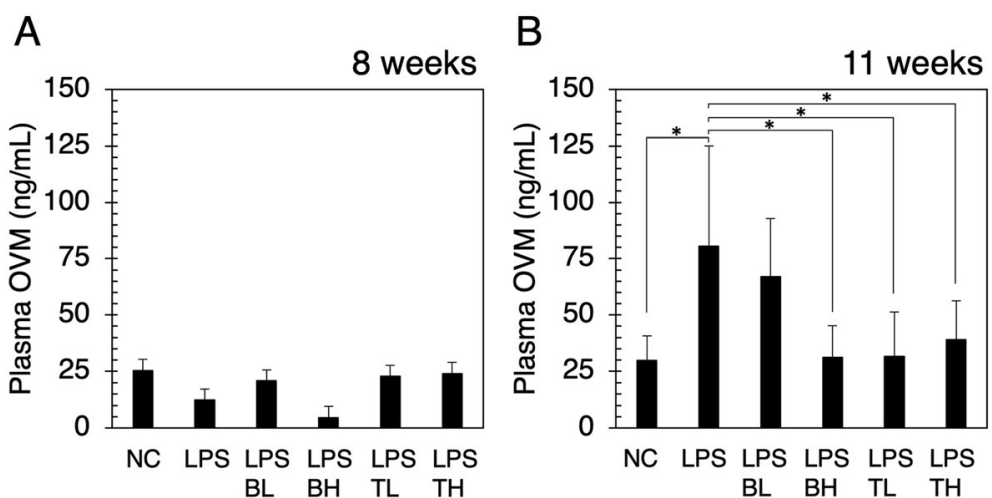

Fig. 2 Detection of plasma OVM after OVM gavage. Mice in OVM group were force fed $0.2 \mathrm{~mL}$ the FITC-labelled OVM (adjusted to $2.5 \mathrm{mg} /$ mouse), $2 \mathrm{~h}$ later blood was collected from saphenous vein at 8 weeks (a) and 11 weeks $(\mathbf{b})(n=6)$. FITC-labelled OVM in plasma was precipitated in $50 \%$ ammonium sulfate and redissolved in water. Fluorescent intensity of sample was measured using fluorescence microplate reader. Differences between groups were considered significantly different when $p<0.05 .{ }^{*} p<0.05$, relative to the LPS group

level was $0.49 \pm 0.23$. Only LPS-BL group $(0.78 \pm 0.12)$ showed increase of the expression, but, LPS-BH $(0.62 \pm$ $0.06)$, LPS-TL $(0.44 \pm 0.15)$ and LPS-TH $(0.28 \pm 0.11)$ group did not increase compared to LPS group. We also compared Cld-2 expression among groups, which is typical of increasing gut permeability. As a result, the value of NC and LPS group were $1.00 \pm 019$ and $1.97 \pm 0.58$, respectively. LPS feeding slightly increased the Cld-2 expression by 2 times and the increase was significantly suppressed in LPS-BL $(0.58 \pm 0.08)$, LPS-BH $(0.63 \pm 0.08)$, LPS-TL $(0.55 \pm 0.11)$ and LPS-TH $(0.55 \pm 0.26)$ groups.

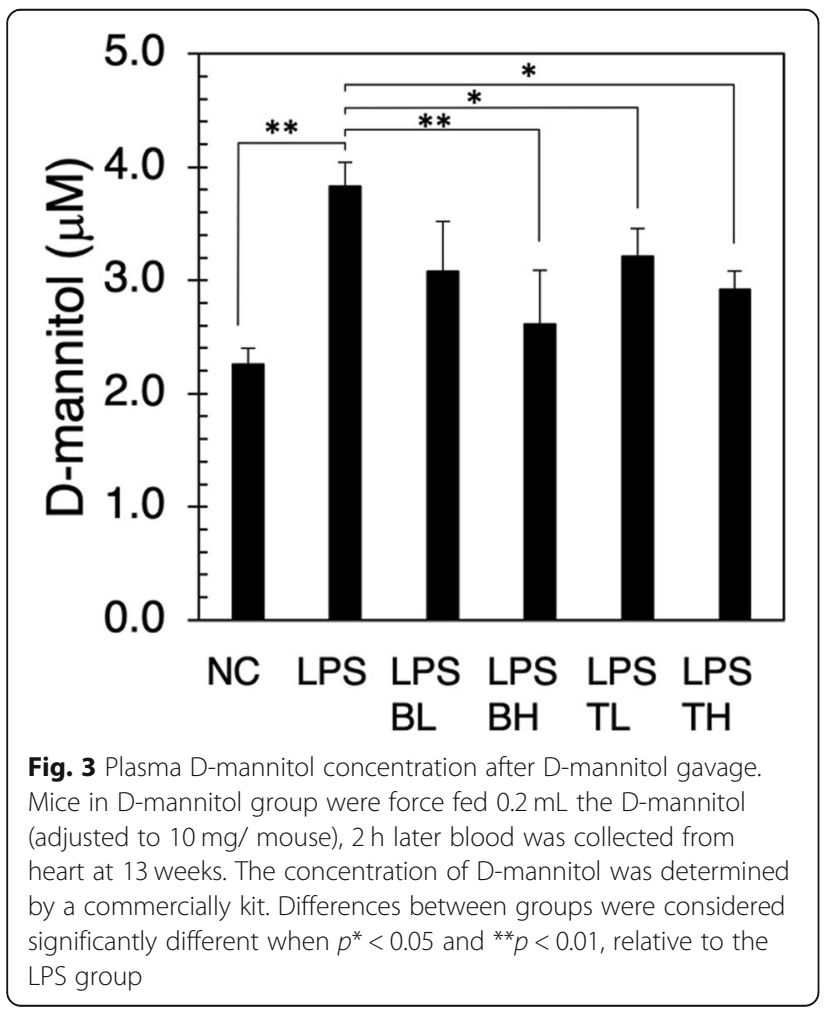

\section{Allergic reactions}

Total IgE concentration and relative specific IgE level against OVM were measured using ELISA (Fig. 5a). Both of them were increased by 1.6-fold in LPS group compared to NC group. Total IgE concentration of each group was $5.00 \pm 1.16,7.97 \pm 0.71,5.20 \pm 0.35,3.12 \pm$ $1.44,8.38 \pm 1.28$, and $5.42 \pm 1.07 \mu \mathrm{g} / \mathrm{mL}$ in NC, LPS, LPS-BL, LPS-BH, LPS-TL, LPS-TH, and LPS-BL group, respectively. Specific IgE level of each group was $1.00 \pm$ $0.03,1.54 \pm 0.20,0.99 \pm 0.03,0.99 \pm 0.02,1.23 \pm 0.15$, and $1.09 \pm 0.14$ in NC, LPS, LPS-BL, LPS-BH, LPS-TL, LPS$\mathrm{TH}$, and LPS-BL group, respectively. Total and specific IgE increased in LPS group. There was significant difference between NC and LPS group in specific IgE. S-PT84 supplementation completely suppressed specific IgE production. RSLE also suppressed specific IgE production in dose-dependent manner. Evaluating allergic reaction by OVM under the LPS feeding, we also measured blood MCPT-1 level using ELISA (Fig. 5b). LPS feeding dramatically increased MCPT-1 concentration by 4.0 -fold. The increase was completely suppressed in LPS-BL and LPS-BH. LPS-TL and LPS also suppressed the increase by LPS, and the effect was dose-dependent.

\section{Discussion}

In the present study, we evaluated whether oral LPS feeding increased gut permeability and triggered an allergic reaction after oral OVM challenge. Wassenaar and Zimmermann (2018) reported that ingested LPS is not toxic because the LPS does not enter the bloodstream. However, our results showed that oral administration of LPS increased gut permeability, and triggered egg allergic reaction by oral challenge of OVM.

We evaluated the gut permeability at 8 weeks and 11 weeks after starting LPS feeding. Eight weeks was not enough to increase permeability in the present conditions. 

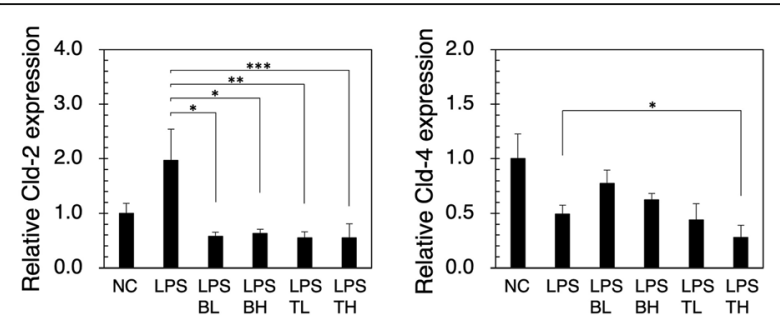

Fig. 4 Semi-quantification of Cld-4 and Cld-2 expression in ileum. Ileum was collected from D-mannitol group $(n=6)$. Cld-4 and Cld-2 expression were evaluated using $2^{-\Delta \Delta C t}$ method. The mean $C t$ value of target genes in the experimental group was normalized to the $\mathrm{Ct}$ value of GAPDH to give a $\Delta C t$ value, which was further normalized to $N C$ group to obtain $\Delta \Delta C$ t. Bars represented the $2^{-\Delta \Delta C t}$ and $R Q$ $\mathrm{min} / \mathrm{max}$ values. $\mathrm{RQ}$ values are derived using standard deviation. Differences between groups were considered significantly different when $p^{*}<0.05,{ }^{* *} p<0.01$ and ${ }^{* * *} p<0.001$ relative to the LPS group

After 11 weeks, LPS treatment increase the plasma OVM concentration after oral challenge, but the results were not identical for all the animals in the same group. At 13 weeks, orally gavage of D-mannitol was significantly high as detected in the blood plasma. We concluded that disturbance of the gut barrier integrity by LPS started after 11th week of treatment and atleast 13 the week of LPS feeding may be required for the apparent increase of gut permeability. Both S-PT84 and RSLE suppressed the increase level of D-mannitol in a dose-dependent manner. Thus, it was suggested that both the S-PT84 and RSLE contributed in maintaining the gut barrier integrity at the presence of LPS.

Craudin- 4 is dominantly expressed in the ileum and belongs as sealing Cld (Garcia-Hernandez et al. 2017). It was suggested that the decrease of Cld-4 by LPS was related to the elevated intestinal permeability. S-PT84 seemed to ameliorate the decrease of Cld- 4 by LPS, but the differences were not significant and did not show dose-dependency. Moreover, RSLE also prevented impaired barrier function, but Cld-4 expression was not influenced in LPS-TL and LPS-TH groups. These results suggest that other molecules cause the prevention of barrier disturbance by S-PT84 and RSLE instead of Cld-4. It has been reported that Cld-2 is highly expressed in leaky epithelia of gastrointestinal inflammation (Luettig et al. 2015). Craudin-2 forms channel for cation and water within T)s (Garcia-Hernandez et al. 2017). Although Cld-2 cannot transport uncharged oligomers such as D-mannitol and LPS, increase of Cld-2 in tight junction could affect the pattern of tight junction strand, resulting in barrier disturbance (Luettig et al. 2015; Liu et al. 2013). Therefore, we also compared the Cld-2 expression among all groups. As a result, LPS slightly increased Cld-2 expression and both S-PT84 and RSLE suppressed the Cld-2 expression. We suspected that the increasing of Cld-2 also related to barrier disturbance but also decreasing of Cld-4. S-PT84 and RSLE may ameliorated the increase of gut permeability via $\mathrm{Cld}-2$ suppression. The underlying protective mechanisms behind the beneficial effect of S-PT84 and RSLE not been fully explored in the present study. However, the beneficial role may be observed due to the impending anti-inflammatory activity of S-PT84 and RSLE or the potential role of S-PT84 and RSLE in modulating the gut microbial community. Therefore, more detailed experiment including protein level evaluations and visualizing of close apposition of membranes using an electron microscope is required (Furuse 2010).

We hypothesized that the increase of gut permeability triggered the allergic response. Specific IgE against OVM and plasma MCPT-1 were significantly increased in LPS feeding group. Plasma MCPT-1 level increase in intestinal immune responses (Miller \& Pemberton 2002). MCPT-1 is predominantly expressed in intestinal mucosal mast cells, and the expression is induced and secreted in the intestinal allergic reaction (Newlands et al. 1987). Therefore, the present results suggested LPS feeding had a potential risk of food allergic reaction. S-PT84 and RSLE decreased the specific IgE and MCPT-1 levels via suppressing of gut permeability. Heat killed S-PT84 induces T helper type 1 dominant state, and regulatory $\mathrm{T}$ cell activity in ovalbumin and alum administrated mice (Nonaka et al. 2008). These results suggest S-PT84 suppresses Th2 effect related to IgE production. RSLE is also suspected of having a suppressive effect of IgE production via NF- $\mathrm{KB}$ suppression (Fan et al. 2015). Therefore, we noted that the effect of S-PT84 and RSLE on IgE production might be caused by not only amelioration of gut inflammation but also anti-allergic reactions.

We suggested that chronic oral LPS feeding increases gut permeability and therefore increases the risk of food allergy. These results were recognized in spite of using the healthy and young mouse that is housed in experimentally controlled environments. It has been reported that gut permeability is increased by alcohol intake (Schaffert et al. 2009; Cho \& Song 2018) and high fat diet (Singh et al. 2017). Gut permeability is also increased in diabetes (Bosi et al. 2006; Cani et al. 2007). These results indicate that increase gut permeability is shown in many people. These people may be exposed by risk of LPS. Recently, it has been reported that gut permeability is related to Alzheimer's disease, systemic lupus erythematosus, and allergic asthma (Obrenovich 2018; Costa et al. 2016; Mu et al. 2015; Farshchi et al. 2017). Therefore, reducing intestinal hyper permeability could ameliorate not only food allergy but also other diseases. Our research suggests that S-PT84 or RSLE could have benefit for health as nutraceuticals.

\section{Conclusions}

Gut inflammation caused by LPS derived from Escherichia coli increased gut permeability and the uptake of 


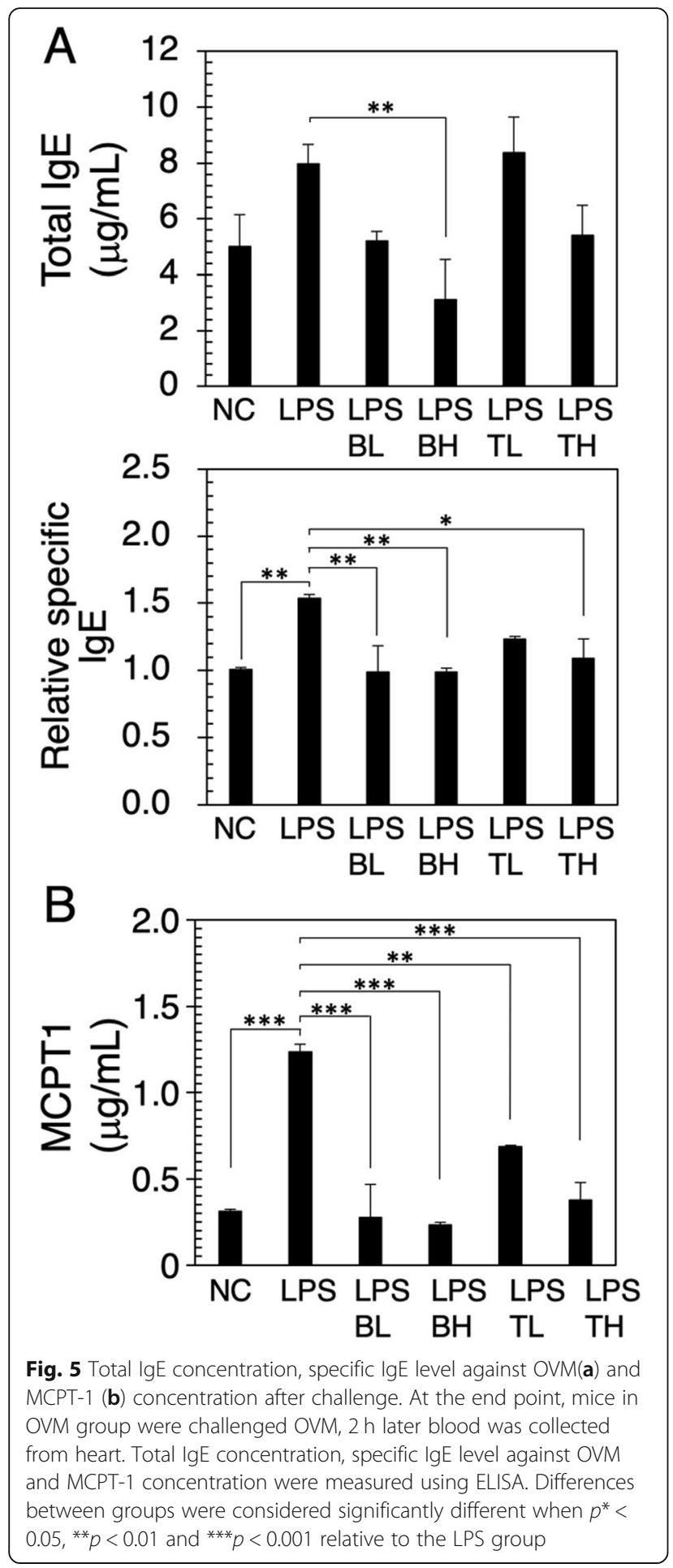

major egg allergen, OVM and resulting in OVMinduced food allergy. However, both the treatment of SPT84 and RSLE suppressed the gut permeability and both treatments also reduced the plasma mast cell protease- 1 and OVM-specific IgE, indicating the potential beneficial effect against LPS intervention developed OVM-induced food allergy.

\section{Supplementary information}

Supplementary information accompanies this paper at https://doi.org/10. 1186/s43014-020-0018-z.

Additional file 1 : Table S1. Composition of experimental foods $(\mathrm{g} / \mathrm{kg})$ simus S. Lee leaf extract.

Additional file 2 : Figure S1. HPLC chromatographic fingerprint of Rubus suavissimus leaf extract.

Additional file 3 : Table S2. Primers used for RT-PCR analysis.

Additional file 4 : Table S3. Fold-changes of tight junction related genes by LPS feeding in jejunum.

\section{Abbreviations}

Cld: Claudins; EGA: Ellagic acid; GA: Gallic acid; LPS: Lipopolysaccharide; NFkB: Nuclear Factor kappa B; OVM: Egg ovomucoid (OVM); RSLE: Rubus suavissimus leaf extract; RUB: Rubusoside; S-PT84: Lactobacillus pentosus SPT84; TJs: Tight junctions; TLR-4: Toll like receptor-4

\section{Acknowledgements}

The authors would like to thank all the staff at the Central Animal facility, University of Guelph and lab colleagues for their skillful technical assistance in help with the animal study.

\section{Authors' contributions}

YM designed and supervised the study and HK, HS and KM performed the experiment and all authors interpreted the data and wrote the paper and agreed to publish.

\section{Funding}

The authors would like to acknowledge funding support to Y. M from NSERC Discovery grant and Kagoshima University Supporting System for Young Faculty Members Overseas Visits Program to HK.

\section{Availability of data and materials}

The datasets generated during and/or analyzed during the current study are available from the corresponding author on reasonable request.

\section{Ethics approval and consent to participate}

All data generated or analyzed during this study were included in this published article.

\section{Competing interests}

The authors declare that they have no competing interests.

\section{Author details}

${ }^{1}$ Department of Food Science, University of Guelph, Guelph, Ontario N1G 2W1, Canada. ${ }^{2}$ Present Address: Department of Clinical Nutrition, Osaka Prefecture University, 3-7-30, Habikino-city, Osaka 583-8555, Japan. ${ }^{3}$ Present Address: Food Science and Technology Department, University of Nebraska, Lincoln, NE 68528, USA. ${ }^{4}$ Institute for Health Care Science, Suntory Wellness Limited, 8-1-1 Seikadai, Seika-cho, Soraku-gun, Kyoto 619-0284, Japan.

Received: 9 August 2019 Accepted: 2 January 2020

Published online: 11 February 2020

\section{References}

Andrade, P. B., \& Valentão, P. (2018). Insights into Natural Products in Inflammation. International Journal of Molecular Sciences. https://doi.org/10. 3390/ijms19030644

Bookout, A. L., \& Mangelsdorf, D. J. (2003). Quantitative real-time PCR protocol for analysis of nuclear receptor signaling pathways. Nuclear Receptor Signaling. https://doi.org/10.1621/nrs.01012.

Bosi, E., Molteni, L., Radaelli, M. G., Folini, L., Fermo, I., Bazzigaluppi, E., et al. (2006). Increased intestinal permeability precedes clinical onset of type 1 diabetes. Diabetologia., 49, 2824-2827.

Cani, P. D., Neyrinck, A. M., Fava, F., Knauf, C., Burcelin, R. G., Tuohy, K. M., et al. (2007). Selective increases of bifidobacteria in gut microflora improve highfat-diet-induced diabetes in mice through a mechanism associated with endotoxaemia. Diabetologia., 50, 2374-2383. 
Chee, M. E., Majumder, K., \& Mine, Y. (2017). Intervention of dietary dipeptide gamma-I-Glutamyl-I-Valine ( $\gamma$-EV) ameliorates inflammatory response in a mouse model of LPS-induced sepsis. Journal of Agricultural and Food Chemistry, 65, 5953-5960.

Cho, Y. E., \& Song, B. J. (2018). Pomegranate prevents binge alcohol-induced gut leakiness and hepatic inflammation by suppressing oxidative and nitrative stress. Redox Biology, 18, 266-278.

Costa, F. R., Françozo, M. C., de Oliveira, G. G., Ignacio, A., Castoldi, A., Zamboni, D. S., et al. (2016). Gut microbiota translocation to the pancreatic lymph nodes triggers NOD2 activation and contributes to T1D onset. The Journal of Experimental Medicine, 213, 1223-1239.

Deitch, E. A., Ma, L., Ma, W. J., Grisham, M. B., Granger, D. N., Specian, R. D., \& Berg, R. D. (1989). Inhibition of endotoxin-induced bacterial translocation in mice. The Journal of Clinical Investigation, 84, 36-42.

Fan, X., Zhang, Y., Dong, H., Wang, B., Ji, H., \& Liu, X. (2015). Trilobatin attenuates the LPS-mediated inflammatory response by suppressing the NF-KB signaling pathway. Food Chemistry, 166, 609-615.

Farshchi, M. K., Azad, F. J., Salari, R., Mirsadraee, M., \& Anushiravani, M. (2017). A viewpoint on the leaky gut syndrome to treat allergic asthma: A novel opinion. Journal of evidence-based complementary \& alternative medicine, 22, 378-380.

Furuse, M. (2010). Molecular basis of the core structure of tight junctions. Cold Spring Harbor Perspectives in Biology. https://doi.org/10.1101/cshperspect.a002907.

Garcia-Hernandez, V., Quiros, M., \& Nusrat, A. (2017). Intestinal epithelial claudins: Expression and regulation in homeostasis and inflammation. Annals of the New York Academy of Sciences, 1397, 66-79.

Kawashima, T., Kosaka, A., Yan, H., Guo, Z., Uchiyama, R., Fukui, R., et al. (2013). Double-stranded RNA of intestinal commensal but not pathogenic bacteria triggers production of protective interferon- $\beta$. Immunity., 38, 1187-1197.

Koh, G. Y., Chou, G., \& Liu, Z. (2009). Purification of a water extract of Chinese sweet tea plant (Rubus suavissimus S. lee) by alcohol precipitation. Journal of Agricultural and Food Chemistry, 57, 5000-5006.

Liu, X., Yang, G., Geng, X. R., Cao, Y., Li, N., Ma, L., et al. (2013). Microbial products induce claudin-2 to compromise gut epithelial barrier function. PLoS One. https://doi.org/10.1371/journal.pone.0068547.

Liu, Y., Alookaran, J. J., \& Rhoads, J. M. (2018). Probiotics in Autoimmune and Inflammatory Disorders. Nutrients. https://doi.org/10.3390/nu10101537.

Lu, Y. C., Yeh, W. C., \& Ohashi, P. S. (2008). LPS/TLR4 signal transduction pathway. Cytokine., 42, 145-151.

Luettig, J., Rosenthal, R., Barmeyer, C., \& Schulzke, J. D. (2015). Claudin-2 as a mediator of leaky gut barrier during intestinal inflammation. Tissue Barriers. https://doi.org/10.4161/21688370.2014.977176.

Maekawa, T., Ishijima, A. S., Ida, M., Izumo, T., Ono, Y., Shibata, H., \& Abe, S. (2016). Prophylactic effect of Lactobacillus pentosus strain S-PT84 on Candida infection and gastric inflammation in a murine gastrointestinal candidiasis model. Medical mycology journal, 57, E81-E92.

Miller, H. R., \& Pemberton, A. D. (2002). Tissue-specific expression of mast cell granule serine proteinases and their role in inflammation in the lung and gut. Immunology., 105, 375-390.

Mu, Q., Kirby, J., Reilly, C. M., \& Luo, X. M. (2017). Leaky gut as a danger signal for autoimmune diseases. Frontiers in Immunology, 8, 598.

Mu, Q., Zhang, H., Liao, X., Lin, K., Liu, H., Edwards, M. R., et al. (2017b). Control of lupus nephritis by changes of gut microbiota. Microbiome., 5, 73.

Mu, Q., Zhang, H., \& Luo, X. M. (2015). SLE: Another autoimmune disorder influenced by microbes and diet? Frontiers in Immunology, 6, 608

Neal, M. D., Sodhi, C. P., Jia, H., Dyer, M., Egan, C. E., Yazji, I., et al. (2012). Toll-like receptor 4 is expressed on intestinal stem cells and regulates their proliferation and apoptosis via the p53 up-regulated modulator of apoptosis The Journal of Biological Chemistry, 287, 37296-37308.

Newlands, G. F., Gibson, S., Knox, D. P., Grencis, R., Wakelin, D., \& Miller, H. R. (1987). Characterization and mast cell origin of a chymotrypsin-like proteinase isolated from intestines of mice infected with Trichinella spiralis. Immunology., 62, 629-634.

Nonaka, Y., Izumo, T., Izumi, F., Maekawa, T., Shibata, H., Nakano, A., et al. (2008). Antiallergic effects of Lactobacillus pentosus strain S-PT84 mediated by modulation of Th1/Th2 immunobalance and induction of IL-10 production. International Archives of Allergy and Immunology, 145, 249-257.

Obrenovich, M. E. M. (2018). Leaky Gut, Leaky Brain? Microorganisms. https://doi. org/10.3390/microorganisms6040107.

Olfert, E. D., Cross, B. M., \& McWilliam, A. A. (2017). Canadian council on animal care. Guide to the care and use of experimental animals (2nd ed.). Ottawa: Canadian Council On Animal Care.
Oz, H. S. (2017). Chronic Inflammatory Diseases and Green Tea Polyphenols. Nutrients. https://doi.org/10.3390/nu9060561.

Ríos-Covián, D., Ruas-Madiedo, P., Margolles, A., Gueimonde, M., de Los ReyesGavilán, C. G., \& Salazar, N. (2016). Intestinal short chain fatty acids and their link with diet and human health. Frontiers in Immunology, 7, 185.

Rupa, P., \& Mine, Y. (2012). Oral immunotherapy with immunodominant T-cell epitope peptides alleviates allergic reactions in a Balb/c mouse model of egg allergy. Allergy., 67, 74-82.

Schaffert, C. S., Duryee, M. J., Hunter, C. D., Hamilton 3rd, B. C., DeVeney, A. L., Huerter, M. M., et al. (2009). Alcohol metabolites and lipopolysaccharide: Roles in the development and/or progression of alcoholic liver disease. World Journal of Gastroenterology, 15, 1209-1218.

Singh, D. P., Singh, J., Boparai, R. K., Zhu, J., Mantri, S., Khare, P., et al. (2017). Isomalto-oligosaccharides, a prebiotic, functionally augment green tea effects against high fat diet-induced metabolic alterations via preventing gut dysbacteriosis in mice. Pharmacological Research, 123, 103-113.

Tamura, S., Shoji, Y., Hasiguchi, K., Aizawa, C., \& Kurata, T. (1994). Effects of cholera toxin adjuvant on IgE antibody response to orally or nasally administered ovalbumin. Vaccine., 12, 1238-1240.

Tsai, Y. L., Lin, T. L., Chang, C. J., Wu, T. R., Lai, W. F., Lu, C. C., \& Lai, H. C. (2019). Probiotics, prebiotics and amelioration of diseases. Journal of Biomedical Science, 26, 3 .

Ukena, S. N., Singh, A., Dringenberg, U., Engelhardt, R., Seidler, U., Hansen, W., et al. (2007). Probiotic Escherichia coli Nissle 1917 inhibits leaky gut by enhancing mucosal integrity. PLoS One. https://doi.org/10.1371/journal.pone.0001308.

Wassenaar, T. M., \& Zimmermann, K. (2018). Lipopolysaccharides in food, food supplements, and probiotics: Should we be worried? European Journal of Microbiology and Immunology, 8, 63-69.

Zhang, Q., Piao, X. L., Piao, X. S., Lu, T., Wang, D., \& Kim, S. W. (2011). Preventive effect of Coptis chinensis and berberine on intestinal injury in rats challenged with lipopolysaccharides. Food and Chemical Toxicology, 49, 61-69.

\section{Publisher's Note}

Springer Nature remains neutral with regard to jurisdictional claims in published maps and institutional affiliations.
Ready to submit your research? Choose BMC and benefit from:

- fast, convenient online submission

- thorough peer review by experienced researchers in your field

- rapid publication on acceptance

- support for research data, including large and complex data types

- gold Open Access which fosters wider collaboration and increased citations

- maximum visibility for your research: over $100 \mathrm{M}$ website views per year

At $\mathrm{BMC}$, research is always in progress.

Learn more biomedcentral.com/submissions 